\title{
A NOTE ON THE MAXIMAL OPERATORS OF VILENKIN-NÖRLUND MEANS WITH NON-INCREASING COEFFICIENTS*
}

\author{
N. MEMIĆ ${ }^{1}$, L. E. PERSSON ${ }^{2,3}$ and G. TEPHNADZE ${ }^{2,4}$ \\ ${ }^{1}$ Department of Mathematics, University of Sarajevo, Zmaja od Bosne 33-35, Sarajevo, \\ Bosnia and Herzegovina \\ e-mail: nacima.o@gmail.com \\ 2 Department of Engineering Sciences and Mathematics, Luleå University of Technology, \\ SE-971 87 Luleå, Sweden \\ e-mail: larserik@ltu.se \\ 3 Narvik University College, P.O. Box 385, N-8505, Narvik, Norway \\ ${ }^{4}$ Department of Mathematics, Faculty of Exact and Natural Sciences, \\ Iv. Javakhishvili Tbilisi State University, Chavchavadze str. 1, Tbilisi 0128, Georgia \\ e-mail: giorgitephnadze@gmail.com
}

Communicated by A. Kroó

(Received March 16, 2015; accepted June 19, 2015)

\section{Abstract}

In [14] we investigated some Vilenkin-Nörlund means with non-increasing coefficients. In particular, it was proved that under some special conditions the maximal operators of such summabily methods are bounded from the Hardy space $H_{1 /(1+\alpha)}$ to the space weak$L_{1 /(1+\alpha)},(0<\alpha \leqq 1)$. In this paper we construct a martingale in the space $H_{1 /(1+\alpha)}$, which satisfies the conditions considered in [14], and so that the maximal operators of these Vilenkin-Nörlund means with non-increasing coefficients are not bounded from the Hardy space $H_{1 /(1+\alpha)}$ to the space $L_{1 /(1+\alpha)}$. In particular, this shows that the conditions under which the result in [14] is proved are in a sense sharp. Moreover, as further applications, some well-known and new results are pointed out.

\section{Introduction and statement of the main result}

Denote by $\mathbb{N}_{+}$the set of the positive integers, $\mathbb{N}:=\mathbb{N}_{+} \cup\{0\}$. Let $m:=$ $\left(m_{0}, m_{1}, \ldots\right)$ be a sequence of the positive integers not less than 2 . Denote by $Z_{m_{k}}:=\left\{0,1, \ldots, m_{k}-1\right\}$ the additive group of integers modulo $m_{k}$.

Define the group $G_{m}$ as the complete direct product of the groups $Z_{m_{i}}$, with the product of the discrete topologies of $Z_{m_{j}}$.

2010 Mathematics Subject Classification. Primary 42C10, 42B25.

Key words and phrases. Vilenkin system, Vilenkin group, Vilenkin-Fourier series, Cesáro means, Fejér means, Nörlund means, martingale Hardy space, $L_{p}$ spaces, weak$L_{p}$ spaces, maximal operator.

${ }^{*}$ The research was supported by a Swedish Institute scholarship, provided within the framework of the SI Baltic Sea Region Cooperation/Visby Programme.

(C) 2016 Akadémiai Kiadó, Budapest 
The direct product $\mu$ of the measures $\mu_{k}(\{j\}):=1 / m_{k}\left(j \in Z_{m_{k}}\right)$ is the Haar measure on $G_{m}$ with $\mu\left(G_{m}\right)=1$.

In this paper we discuss bounded Vilenkin groups, i.e. the case when $\sup _{n} m_{n}<\infty$.

The elements of $G_{m}$ are represented by sequences $x:=\left(x_{0}, x_{1}, \ldots, x_{j}, \ldots\right)$ $\left(x_{j} \in Z_{m_{j}}\right)$.

It is easy to give a base for the neighborhoods of $G_{m}$ :

$$
I_{0}(x):=G_{m}, \quad I_{n}(x):=\left\{y \in G_{m} \mid y_{0}=x_{0}, \ldots, y_{n-1}=x_{n-1}\right\},
$$

where $x \in G_{m}, n \in \mathbb{N}$. Denote $I_{n}:=I_{n}(0)$ for $n \in \mathbb{N}_{+}$, and $\overline{I_{n}}:=G_{m} \backslash I_{n}$.

If we define the so-called generalized number system based on $m$ in the following way:

$$
M_{0}:=1, \quad M_{k+1}:=m_{k} M_{k} \quad(k \in \mathbb{N}),
$$

then every $n \in \mathbb{N}$ can be uniquely expressed as $n=\sum_{j=0}^{\infty} n_{j} M_{j}$, where $n_{j} \in Z_{m_{j}}\left(j \in \mathbb{N}_{+}\right)$and only a finite number of $n_{j}{ }^{6}$ s differ from zero.

Next, we introduce on $G_{m}$ an orthonormal system which is called the Vilenkin system. At first, we define the complex-valued function $r_{k}(x)$ : $G_{m} \rightarrow \mathbb{C}$, the generalized Rademacher functions, by

$$
r_{k}(x):=\exp \left(2 \pi i x_{k} / m_{k}\right), \quad\left(i^{2}=-1, x \in G_{m}, k \in \mathbb{N}\right) .
$$

Now, define the Vilenkin system $\psi:=\left(\psi_{n}: n \in \mathbb{N}\right)$ on $G_{m}$ as:

$$
\psi_{n}(x):=\prod_{k=0}^{\infty} r_{k}^{n_{k}}(x), \quad(n \in \mathbb{N}) .
$$

Specifically, we call this system the Walsh-Paley system when $m \equiv 2$.

The Vilenkin system is orthonormal and complete in $L_{2}\left(G_{m}\right)$ (see [17]).

The norm (or quasi-norm) of the space $L_{p}\left(G_{m}\right)$ and weak- $L_{p}\left(G_{m}\right)$ $(0<p<\infty)$ are respectively defined by

$$
\|f\|_{p}^{p}:=\int_{G_{m}}|f|^{p} d \mu, \quad\|f\|_{\text {weak- } L_{p}}^{p}:=\sup _{\lambda>0} \lambda^{p} \mu(f>\lambda)<+\infty .
$$

If $f \in L_{1}\left(G_{m}\right)$ we can respectively define the Fourier coefficients, the partial sums of the Fourier series, the Dirichlet kernels with respect to the Vilenkin system in the usual manner:

$$
\widehat{f}(n):=\int_{G_{m}} f \bar{\psi}_{n} d \mu, \quad(n \in \mathbb{N}),
$$




$$
S_{n} f:=\sum_{k=0}^{n-1} \widehat{f}(k) \psi_{k}, \quad D_{n}:=\sum_{k=0}^{n-1} \psi_{k}, \quad\left(n \in \mathbb{N}_{+}\right)
$$

Recall that

$$
D_{M_{n}}(x)= \begin{cases}M_{n}, & \text { if } x \in I_{n}, \\ 0, & \text { if } x \notin I_{n} .\end{cases}
$$

The $\sigma$-algebra generated by the intervals $\left\{I_{n}(x): x \in G_{m}\right\}$ will be denoted by $\digamma_{n}(n \in \mathbb{N})$. Denote by $f=\left(f^{(n)}, n \in \mathbb{N}\right)$ a martingale with respect to $\digamma_{n}(n \in \mathbb{N})$. (for details see e.g. [18]).

The maximal function of a martingale $f$ is defined by

$$
f^{*}=\sup _{n \in \mathbb{N}}\left|f^{(n)}\right| .
$$

For $0<p<\infty$ the Hardy martingale spaces $H_{p}\left(G_{m}\right)$ consist of all martingales for which

$$
\|f\|_{H_{p}}:=\left\|f^{*}\right\|_{p}<\infty
$$

If $f=\left(f^{(n)}, n \in \mathbb{N}\right)$ is a martingale, then the Vilenkin-Fourier coefficients must be defined in a slightly different manner:

$$
\widehat{f}(i):=\lim _{k \rightarrow \infty} \int_{G_{m}} f^{(k)} \bar{\psi}_{i} d \mu .
$$

A bounded measurable function $a$ is a p-atom $(p>0)$, if there exists an interval $I$, such that

$$
\int_{I} a d \mu=0, \quad\|a\|_{\infty} \leqq \mu(I)^{-1 / p}, \quad \operatorname{supp}(a) \subset I .
$$

We also need the following auxiliary result (see [19]):

Lemma 1. A martingale $f=\left(f^{(n)}, n \in \mathbb{N}\right)$ is in $H_{p}(0<p \leqq 1)$ if and only if there exists a sequence $\left(a_{k}, k \in \mathbb{N}\right)$ of $p$-atoms and a sequence $\left(\mu_{k}, k \in \mathbb{N}\right)$ of real numbers, such that, for every $n \in \mathbb{N}$,

$$
\sum_{k=0}^{\infty} \mu_{k} S_{M_{n}} a_{k}=f^{(n)}, \quad \sum_{k=0}^{\infty}\left|\mu_{k}\right|^{p}<\infty .
$$


Moreover, $\|f\|_{H_{p}} \backsim \inf \left(\sum_{k=0}^{\infty}\left|\mu_{k}\right|^{p}\right)^{1 / p}$, where the infimum is taken over all decompositions of $f$ of the form (2).

Let $\left\{q_{k}: k \geqq 0\right\}$ be a sequence of nonnegative numbers. The $n$-th Nörlund mean for a Fourier series of $f$ is defined by

$$
t_{n} f=\frac{1}{Q_{n}} \sum_{k=1}^{n} q_{n-k} S_{k} f
$$

where $Q_{n}:=\sum_{k=0}^{n-1} q_{k}$.

We always assume that $q_{0}>0$ and $\lim _{n \rightarrow \infty} Q_{n}=\infty$. In this case it is well-known that the summability method generated by $\left\{q_{k}: k \geqq 0\right\}$ is regular if and only if

$$
\lim _{n \rightarrow \infty} \frac{q_{n-1}}{Q_{n}}=0 .
$$

Concerning this fact and related basic results, we refer to [6].

The $(C, \alpha)$-means (Cesáro means) of the Vilenkin-Fourier series are defined by

$$
\sigma_{n}^{\alpha} f=\frac{1}{A_{n}^{\alpha}} \sum_{k=1}^{n} A_{n-k}^{\alpha-1} S_{k} f
$$

where

$$
A_{0}^{\alpha}=0, \quad A_{n}^{\alpha}=\frac{(\alpha+1) \ldots(\alpha+n)}{n !}, \quad \alpha \neq-1,-2, \ldots
$$

When $\alpha=1$ the Cesáro means coincide with the Fejér means

$$
\sigma_{n} f=\frac{1}{n} \sum_{k=1}^{n} S_{k} f
$$

For the martingale $f$ we consider the following maximal operators:

$$
t^{*} f:=\sup _{n \in \mathbb{N}}\left|t_{n} f\right|, \quad \sigma^{*} f:=\sup _{n \in \mathbb{N}}\left|\sigma_{n} f\right|, \quad \sigma^{\alpha, *} f:=\sup _{n \in \mathbb{N}}\left|\sigma_{n}^{\alpha} f\right| .
$$

In the one-dimensional case the result with respect to the a.e. convergence of Fejér is due to Pál and Simon [11] (c.f. also [2]) for bounded Vilenkin series. Weisz [20] proved that the maximal operator of the Fejér means $\sigma^{*}$ is bounded from the Hardy space $H_{1 / 2}$ to the space weak- $L_{1 / 2}$. Simon [12] gave a counterexample, which shows that boundedness does not hold for $0<p<1 / 2$. A counterexample for $p=1 / 2$ was given in [16].

In [4] Goginava investigated the behaviour of Cesáro means of WalshFourier series in detail. The a.e. convergence of Cesáro means of $f \in L_{1}$ was proved in [5]. Furthermore, Simon and Weisz [13] showed that the maximal 
operator $\sigma^{\alpha, *}(0<\alpha<1)$ of the $(C, \alpha)$ means is bounded from the Hardy space $H_{1 /(1+\alpha)}$ to the space weak- $L_{1 /(1+\alpha)}$. Moreover, Goginava [3] gave a counterexample, which shows that boundedness does not hold for $0<p \leqq$ $1 /(1+\alpha)$.

Móricz and Siddiqi [7] investigated the approximation properties of some special Nörlund means of Walsh-Fourier series of $L_{p}$ functions in norm. In the two-dimensional case approximation properties of Nörlund was considered by Nagy (see [8]-[10]). In [1] and [15] it was proved strong convergence theorems for Nörlund means of Vilenkin-Fourier series with monotone coefficients. Moreover, there was also shown boundedness of weighted maximal operators of such Nörlund means on martingale Hardy spaces. Recently, in [14] it was proved that the following is true:

Theorem A. a) Let $0<\alpha \leqq 1$. Then the maximal operator $t^{*}$ of summability method (3) with non-increasing sequence $\left\{q_{k}: k \geqq 0\right\}$, satisfying the conditions

$$
\frac{n^{\alpha} q_{0}}{Q_{n}}=O(1), \quad \frac{\left|q_{n}-q_{n+1}\right|}{n^{\alpha-2}}=O(1), \quad \text { as } n \rightarrow \infty,
$$

is bounded from the Hardy space $H_{1 /(1+\alpha)}$ to the space weak- $L_{1 /(1+\alpha)}$.

b) Let $0<\alpha \leqq 1,0 \leqq p<1 /(1+\alpha)$ and $\left\{q_{k}: k \geqq 0\right\}$ be a non-increasing sequence, satisfying the condition

$$
\frac{q_{0}}{Q_{n}} \geqq \frac{c}{n^{\alpha}}, \quad(c>0) .
$$

Then there exists a martingale $f \in H_{p}$, such that

$$
\sup _{n \in \mathbb{N}}\left\|t_{n} f\right\|_{\text {weak- } L_{p}}=\infty \text {. }
$$

c) Let $\left\{q_{k}: k \geqq 0\right\}$ be a non-increasing sequence, satisfying the condition

$$
\varlimsup_{n \rightarrow \infty} \frac{q_{0} n^{\alpha}}{Q_{n}}=\infty, \quad(0<\alpha \leqq 1) .
$$

Then there exists an martingale $f \in H_{1 /(1+\alpha)}$, such that

$$
\sup _{n \in \mathbb{N}}\left\|t_{n} f\right\|_{\text {weak-L } L_{1 /(1+\alpha)}}=\infty \text {. }
$$

In this paper we complement this result by proving sharpness of both conditions of (4). Our main result reads: 
THEOREM 1. Let $0<\alpha \leqq 1$ and $\left\{q_{k}: k \geqq 0\right\}$ be a non-increasing sequence, satisfying the conditions

$$
\varlimsup_{n \rightarrow \infty} \frac{\left|q_{n}-q_{n+1}\right|}{n^{\alpha-2}} \geqq c, \quad(c>0),
$$

and

$$
\frac{n^{\alpha} q_{0}}{Q_{n}} \geqq c, \quad(c>0, n \in \mathbb{N}) .
$$

Then there exists a martingale $f \in H_{1 /(1+\alpha)}$, such that

$$
\sup _{n \in \mathbb{N}}\left\|t_{n} f\right\|_{1 /(1+\alpha)}=\infty \text {. }
$$

The proof can be found in the Section 2 and some applications and final remark in the Section 3.

\section{Proof of Theorem 1}

Proof. Under the condition (7), there exists an increasing sequence $\left\{n_{k}: k \in \mathbb{N}\right\}$ of positive integers such that

$$
\frac{M_{2 n_{k}+1}^{\alpha}}{Q_{M_{2 n_{k}}+1}}>c_{\alpha}>0, \quad k \in \mathbb{N} .
$$

Let $\left\{\alpha_{k}: k \in \mathbb{N}\right\} \in\left\{n_{k}: k \in \mathbb{N}\right\}$ be an increasing sequence of positive integers such that:

$$
\begin{gathered}
\sum_{k=0}^{\infty} 1 / \alpha_{k}^{1 /(1+\alpha)}<\infty \\
\lambda \sum_{\eta=0}^{k-1} \frac{M_{\alpha_{\eta}}^{1+\alpha}}{\alpha_{\eta}}<\frac{M_{\alpha_{k}}^{1+\alpha}}{\alpha_{k}}
\end{gathered}
$$

and

$$
\frac{32 \lambda M_{\alpha_{k-1}}^{1+\alpha}}{\alpha_{k-1}}<\frac{M_{\left[\alpha_{k} / 2\right]}^{\alpha+1}}{\alpha_{k}},
$$


where $\lambda=\sup _{n} m_{n}$ and $\left[\alpha_{k} / 2\right]$ denotes the integer part of $\alpha_{k} / 2$.

We note that such increasing sequence $\left\{\alpha_{k}: k \in \mathbb{N}\right\}$ which satisfies conditions (10)-(12) can be constructed.

Let the martingale $f:=\left(f^{(n)}: n \in \mathbb{N}\right)$ be defined by

$$
f^{(n)}=\sum_{\left\{k: \alpha_{k}<n\right\}} \lambda_{k} \theta_{\alpha_{k}},
$$

where

$$
\lambda_{k}=\frac{\lambda}{\alpha_{k}} \quad \text { and } \quad \theta_{\alpha_{k}}=\frac{M_{\alpha_{k}}^{\alpha}}{\lambda}\left(D_{M_{\alpha_{k}+1}}-D_{M_{\alpha_{k}}}\right) .
$$

Since

$$
\begin{aligned}
S_{M_{A}} \theta_{k} & = \begin{cases}\theta_{k}, & \text { if } \alpha_{k}<A, \\
0, & \text { if } \alpha_{k} \geqq A,\end{cases} \\
\operatorname{supp}\left(\theta_{k}\right) & =I_{\alpha_{k}}, \quad \int_{I_{\alpha_{k}}} \theta_{k} d \mu=0, \quad\left\|\theta_{k}\right\|_{\infty} \leqq M_{\alpha_{k}}^{1+\alpha}=\left(\operatorname{supp} \theta_{k}\right)^{1+\alpha},
\end{aligned}
$$

if we apply Lemma 1 and (10) we can conclude that $f \in H_{1 /(1+\alpha)}$.

Moreover, it is easy to see that

$$
\widehat{f}(j)= \begin{cases}\frac{M_{\alpha_{k}}^{\alpha},}{\alpha_{k}}, & \text { if } j \in\left\{M_{\alpha_{k}}, \ldots, M_{\alpha_{k}+1}-1\right\}, k=0,1,2 \ldots, \\ 0, & \text { if } j \notin \bigcup_{k=1}^{\infty}\left\{M_{\alpha_{k}}, \ldots, M_{\alpha_{k}+1}-1\right\} .\end{cases}
$$

Let $s=0, \ldots, k-1$. We can write that

$$
\begin{aligned}
& t_{M_{\alpha_{k}}+M_{s}} f \\
= & \frac{1}{Q_{M_{\alpha_{k}}+M_{s}}} \sum_{j=0}^{M_{\alpha_{k}}} q_{j} S_{j} f+\frac{1}{Q_{M_{\alpha_{k}}+M_{s}}} \sum_{j=M_{\alpha_{k}}+1}^{M_{\alpha_{k}}+M_{s}} q_{j} S_{j} f \\
:= & I+I I .
\end{aligned}
$$

Let $M_{\alpha_{s}} \leqq j \leqq M_{\alpha_{s}+1}$, where $s=0, \ldots, k-1$. Moreover,

$$
\left|D_{j}-D_{M_{\alpha_{s}}}\right| \leqq 2 j \leqq \lambda M_{\alpha_{s}}, \quad(s \in \mathbb{N})
$$


so that, according to (1) and (15), we have that

$(16)$

$$
\begin{aligned}
& \left|S_{j} f\right| \\
= & \left|\sum_{v=0}^{M_{\alpha_{s-1}+1}-1} \widehat{f}(v) \psi_{v}+\sum_{v=M_{\alpha_{s}}}^{j-1} \widehat{f}(v) \psi_{v}\right| \\
\leqq & \left|\sum_{\eta=0}^{s-1} \sum_{v=M_{\alpha_{\eta}}}^{M_{\alpha_{\eta}+1}-1} \frac{M_{\alpha_{\eta}}^{\alpha}}{\alpha_{\eta}} \psi_{v}\right| \\
& +\frac{M_{\alpha_{s}}^{\alpha}}{\alpha_{s}}\left|\left(D_{j}-D_{M_{\alpha_{s}}}\right)\right| \\
= & \left|\sum_{\eta=0}^{s-1} \frac{M_{\alpha_{\eta}}^{\alpha}}{\alpha_{\eta}}\left(D_{M_{\alpha_{\eta}+1}}-D_{M_{\alpha_{\eta}}}\right)\right| \\
& +\frac{M_{\alpha_{s}}^{\alpha}}{\alpha_{s}}\left|\left(D_{j}-D_{M_{\alpha_{s}}}\right)\right| \\
\leqq & \lambda \sum_{\eta=0}^{s-1} \frac{M_{\alpha_{\eta}}^{\alpha+1}}{\alpha_{\eta}}+\frac{\lambda M_{\alpha_{s}}^{\alpha+1}}{\alpha_{s}} \\
\leqq & \frac{\lambda M_{\alpha_{s}}^{\alpha+1}}{\alpha_{s}}+\frac{\lambda M_{\alpha_{s}}^{\alpha+1}}{\alpha_{s}} \leqq \frac{2 \lambda M_{\alpha_{k-1}}^{\alpha+1}}{\alpha_{k-1}} .
\end{aligned}
$$

Let $M_{\alpha_{s-1}+1}+1 \leqq j \leqq M_{\alpha_{s}}$, where $s=1, \ldots, k$. Analogously to (16) we find that

$$
\begin{aligned}
& \left|S_{j} f\right|=\left|\sum_{v=0}^{M_{\alpha_{s-1}+1}-1} \widehat{f}(v) \psi_{v}\right|=\left|\sum_{\eta=0}^{s-1} \sum_{v=M_{\alpha_{\eta}}}^{M_{\alpha_{\eta}+1}-1} \frac{M_{\alpha_{\eta}}^{\alpha}}{\alpha_{\eta}} \psi_{v}\right| \\
= & \left|\sum_{\eta=0}^{s-1} \frac{M_{\alpha_{\eta}}^{\alpha}}{\alpha_{\eta}}\left(D_{M_{\alpha_{\eta}+1}}-D_{M_{\alpha_{\eta}}}\right)\right| \leqq \frac{2 \lambda M_{\alpha_{k-1}}^{\alpha+1}}{\alpha_{k-1}} .
\end{aligned}
$$

Hence,

$$
|I| \leqq \frac{1}{Q_{M_{\alpha_{k}}+M_{s}}} \sum_{j=0}^{M_{\alpha_{k}}} q_{j}\left|S_{j} f\right|
$$




$$
\begin{aligned}
& \leqq \frac{2 \lambda M_{\alpha_{k-1}}^{\alpha+1}}{\alpha_{k-1}} \frac{1}{Q_{M_{\alpha_{k}}+M_{s}}} \sum_{j=0}^{M_{\alpha_{k}}} q_{j} \\
& \leqq \frac{2 \lambda M_{\alpha_{k-1}}^{\alpha+1}}{\alpha_{k-1}}
\end{aligned}
$$

Let $x \in I_{s} / I_{s+1}$. Since

(18) $D_{j+M_{n}}=D_{M_{n}}+\psi_{M_{n}} D_{j}=D_{M_{n}}+r_{n} D_{j}$, when $j<M_{n}$,

if we now apply Abel transformation, (15) and inequalities of (8) and (9) we get that

$$
\begin{aligned}
|I I| & =\frac{1}{Q_{M_{\alpha_{k}}+M_{s}}}\left|\frac{M_{\alpha_{k}}^{\alpha}}{\alpha_{k}} \sum_{j=M_{\alpha_{k}}+1}^{M_{\alpha_{k}}+M_{s}} q_{M_{\alpha_{k}}+M_{s}-j}\left(D_{j}-D_{M_{\alpha_{k}}}\right)\right| \\
& =\frac{1}{Q_{M_{\alpha_{k}}+M_{s}}}\left|\frac{M_{\alpha_{k}}^{\alpha}}{\alpha_{k}} \sum_{j=1}^{M_{s}} q_{M_{s}-j}\left(D_{j+M_{\alpha_{k}}}-D_{M_{\alpha_{k}}}\right)\right| \\
& =\frac{1}{Q_{M_{\alpha_{k}}+M_{s}}}\left|\frac{\psi_{M_{\alpha_{k}}} M_{\alpha_{k}}^{\alpha}}{\alpha_{k}} \sum_{j=1}^{M_{s}} q_{M_{s}-j} D_{j}\right| \\
& =\frac{M_{\alpha_{k}}^{\alpha}}{\alpha_{k} Q_{M_{\alpha_{k}}+M_{s}}}\left|\sum_{j=1}^{M_{s}} q_{M_{s}-j j}\right| \\
& \geqq \frac{c}{\alpha_{k}}\left|\sum_{j=1}^{M_{s}}\left(q_{M_{s}-j}-q_{M_{s}-j-1}\right) j^{2}\right| \\
& \geqq \frac{c M_{s}^{2}}{\alpha_{k}} \sum_{j=\left[M_{s} / 2\right]}^{M_{s}}\left|q_{M_{s}-j}-q_{M_{s}-j-1}\right| \\
& \geqq \frac{c M_{s}^{2}}{\alpha_{k}} \sum_{j=0}^{\left[M_{s} / 2\right]}\left|q_{j}-q_{j+1}\right| \\
& \geqq \frac{c M_{s}^{2}}{\alpha_{k}} \sum_{j=0}^{\left[M_{s} / 2\right]} j^{\alpha-2}
\end{aligned}
$$




$$
\geqq \frac{c M_{s}^{\alpha-1} M_{s}^{2}}{\alpha_{k}} \geqq \frac{c M_{s}^{\alpha+1}}{\alpha_{k}} .
$$

Let $\left[\alpha_{k} / 2\right]<s \leqq \alpha_{k}$. Therefore, it yields that

$$
\begin{gathered}
\int_{G_{m}}\left|t_{M_{\alpha_{k}}+M_{s}} f(x)\right|^{1 /(1+\alpha)} d \mu(x) \\
\geqq|I I|-|I| \geqq \frac{c M_{s}^{1+\alpha}}{\alpha_{k}}-\frac{4 \lambda M_{\alpha_{k-1}}^{\alpha+1}}{\alpha_{k-1}} \geqq \frac{c M_{s}^{1+\alpha}}{\alpha_{k}} .
\end{gathered}
$$

By combining (17) and (19) we get that

$$
\begin{aligned}
& \int_{G_{m}}\left|t^{*} f\right|^{1 /(1+\alpha)} d \mu \\
\geqq & \sum_{s=\left[\alpha_{k} / 2\right]+1}^{\alpha_{k}} \int_{I_{s} / I_{s+1}}\left|t_{M_{\alpha_{k}}+M_{s}} f\right|^{1 /(1+\alpha)} d \mu \\
\geqq & c \sum_{s=\left[\alpha_{k} / 2\right]}^{\alpha_{k}} \frac{M_{s}}{M_{s} \alpha_{k}^{1 /(1+\alpha)} \geqq c \sum_{s=\left[\alpha_{k} / 2\right]}^{\alpha_{k}-3} \frac{1}{\alpha_{k}^{1 /(1+\alpha)}}} \\
\geqq & \frac{c}{\alpha_{k}^{1 /(1+\alpha)}} \sum_{s=\left[\alpha_{k} / 2\right]}^{\alpha_{k}} 1 \geqq \frac{c \alpha_{k}}{\alpha_{k}^{1 /(1+\alpha)}} \\
\geqq & c \alpha_{k}^{\alpha /(1+\alpha)} \rightarrow \infty, \quad \text { as } k \rightarrow \infty .
\end{aligned}
$$

The proof is complete.

\section{Applications and final remark}

REMARK 1 . We note that under the both conditions of (7) in Theorem 1 the conditions (4) in Theorem A can still be fulfilled. So our main result shows that under the both conditions of (7) in part a) of Theorem A are in a sence sharp and the point $p=1 /(1+\alpha)$ is the smallest number for which we have boundedness from the Hardy space $H_{1 /(1+\alpha)}$ to the space weak$L_{1 /(1+\alpha)}$. 
Our main result Theorem 1 immediately implies the following results of Goginava [3] and Tephnadze [16]:

Corollary 1 (Goginava). The maximal operator of the $(C, \alpha)$-means $\sigma^{\alpha, *}$ is not bounded from the Hardy space $H_{1 /(1+\alpha)}$ to the space $L_{1 /(1+\alpha)}$, where $0<\alpha \leqq 1$.

Corollary 2 (Tephnadze). The maximal operator of the Fejér means $\sigma^{*}$ is not bounded from the Hardy space $H_{1 / 2}$ to the space $L_{1 / 2}$. is

Let $\theta_{n}^{\alpha}$ denote the Nörlund mean, where $\left\{q_{0}=0, q_{k}=k^{\alpha-1}: k \geqq 1\right\}$, that

$$
\theta_{n}^{\alpha} f=\frac{1}{Q_{n}} \sum_{k=1}^{n}(n-k)^{\alpha-1} S_{k} f
$$

It is easy to see that

$$
\begin{aligned}
& \frac{\left|q_{n}-q_{n+1}\right|}{n^{\alpha-2}}=\frac{1}{n^{\alpha-2}}\left(\frac{n^{\alpha}}{n}-\frac{(n+1)^{\alpha}}{n+1}\right) \\
\leqq & \frac{1}{n^{\alpha-2}}\left(\frac{n^{\alpha}}{n}-\frac{n^{\alpha}}{n+1}\right)=\frac{1}{n^{\alpha-2}} \frac{n^{\alpha}}{n(n+1)} \\
\leqq & \frac{1}{n^{\alpha-2}} \frac{2}{n^{2-\alpha}}=O(1), \quad \text { as } n \rightarrow \infty .
\end{aligned}
$$

Since

$$
Q_{n}:=\sum_{k=0}^{n-1} k^{\alpha-1} \geqq \int_{1}^{n-1} x^{\alpha-1} d x \geqq c n^{\alpha}
$$

we obtain that

$$
\frac{n^{\alpha} q_{0}}{Q_{n}}=O(1), \quad \text { as } n \rightarrow \infty
$$

By combining inequalities (20) and (21) we get the following new result:

COROLlary 3. The maximal operator of the $\theta_{n}^{\alpha}$-means

$$
\theta^{\alpha, *}:=\sup _{n \in \mathbb{N}}\left|\theta_{n} f\right|
$$

is not bounded from the martingale Hardy space $H_{1 /(1+\alpha)}$ to the Lebesgue space $L_{1 /(1+\alpha)}$, where $0<\alpha \leqq 1$. 
Acknowledgment. We thank the referee for some valuable remarks, which have improved the final version of this paper.

\section{REFERENCES}

[1] Blahota, I., Persson, L. E. and Tephnadze, G., On the Nörlund means of Vilenkin-Fourier series, Czechoslovak Math. J. (to appear).

[2] GÁt, G., Cesàro means of integrable functions with respect to unbounded Vilenkin systems, J. Approx. Theory, 124 (2003), no. 1, 25-43.

[3] Goginava, U., The maximal operator of the $(C, \alpha)$ means of the Walsh-Fourier series, Ann. Univ. Sci. Budapest. Sect. Comput., 26 (2006), 127-135.

[4] Goginava, U., On the approximation properties of Cesáro means of negative order of Walsh-Fourier series, J. Approx. Theory, 115 (2002), no. 1, 9-20.

[5] Goginava, U., Almost everywhere convergence of $(C, \alpha)$-means of cubical partial sums of d-dimensional Walsh-Fourier series, J. Approx. Theory, 141 (2006), no. $1,8-28$.

[6] Moore, C. N., Summable series and convergence factors, Dover Publications, Inc., New York, 1966.

[7] Móricz, F. and Siddiqi, A., Approximation by Nörlund means of Walsh-Fourier series, J. Approx. Theory, 70 (1992), no. 3, 375-389.

[8] Nagy, K., Approximation by Nörlund means of Walsh-Kaczmarz-Fourier series, Georgian Math. J., 18 (2011), no. 1, 147-162.

[9] NAGY, K., Approximation by Nörlund means of quadratical partial sums of double Walsh-Fourier series, Anal. Math., 36 (2010), no. 4, 299-319.

[10] Nagy, K., Approximation by Nörlund means of double Walsh-Fourier series for Lipschitz functions, Math. Inequal. Appl., 15 (2012), no. 2, 301-322.

[11] PÁL, J. and P. Simon, On a generalization of the concept of derivative, Acta Math. Acad. Sci. Hungar. 29 (1977), no. 1-2, 155-164.

[12] Simon, P., Cesáro summability with respect to two-parameter Walsh systems, Monatsh. Math., 131 (2000), no. 4, 321-334.

[13] Simon, P. and Weisz, F., Weak inequalities for Cesáro and Riesz summability of Walsh-Fourier series, J. Approx. Theory, 151 (2008), no. 1, 1-19.

[14] Persson, L.-E., Tephnadze, G. and Wall, P., On the maximal operators of Vilenkin-Nörlund means, J. Fourier Anal. Appl., 21 (2015), no. 1, 76-94.

[15] Persson, L.-E., Tephnadze, G. and Wall, P., Some new $\left(H_{p}, L_{p}\right)$ type inequalities of maximal operators of Vilenkin-Nörlund means with non-decreasing coefficients, J. Math. Inequal. (to appear).

[16] Tephnadze, G., Fejér means of Vilenkin-Fourier series, Studia Sci. Math. Hungar., 49 (2012), no. 1, 79-90.

[17] Vilenkin, N., On a class of complete orthonormal systems, Amer. Math. Soc. Transl., 28 (1963), no. 2, 1-35.

[18] Weisz, F., Martingale Hardy spaces and their applications in Fourier analysis, Lecture Notes in Mathematics, 1568, Springer-Verlag, Berlin, 1994.

[19] Weisz, F., Summability of multi-dimensional Fourier series and Hardy space, Kluwer Academic, Dordrecht, 2002.

[20] Weisz, F., Cesáro summability of one- and two-dimensional Walsh-Fourier series, Anal. Math., 22 (1996), no. 3, 229-242. 